\title{
Potential Use of a Serpin from Arabidopsis for Pest Control
}

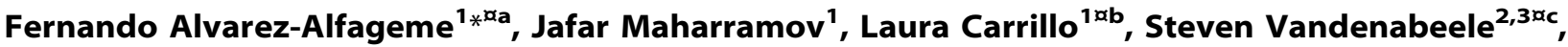 \\ Dominique Vercammen $^{2,3 x d}$, Frank Van Breusegem ${ }^{2,3}$, Guy Smagghe ${ }^{1 *}$
}

1 Laboratory of Agrozoology, Department of Crop Protection, Faculty of Bioscience Engineering, Ghent University, Gent, Belgium, 2 VIB Department of Plant Systems Biology, Ghent University, Gent, Belgium, 3 Department of Plant Biotechnology and Genetics, Ghent University, Gent, Belgium

\begin{abstract}
Although genetically modified (GM) plants expressing toxins from Bacillus thuringiensis (Bt) protect agricultural crops against lepidopteran and coleopteran pests, field-evolved resistance to $B t$ toxins has been reported for populations of several lepidopteran species. Moreover, some important agricultural pests, like phloem-feeding insects, are not susceptible to $B t$ crops. Complementary pest control strategies are therefore necessary to assure that the benefits provided by those insectresistant transgenic plants are not compromised and to target those pests that are not susceptible. Experimental GM plants producing plant protease inhibitors have been shown to confer resistance against a wide range of agricultural pests. In this study we assessed the potential of AtSerpin1, a serpin from Arabidopsis thaliana (L). Heynh., for pest control. In vitro assays were conducted with a wide range of pests that rely mainly on either serine or cysteine proteases for digestion and also with three non-target organisms occurring in agricultural crops. AtSerpin 1 inhibited proteases from all pest and non-target species assayed. Subsequently, the cotton leafworm Spodoptera littoralis Boisduval and the pea aphid Acyrthosiphon pisum (Harris) were fed on artificial diets containing AtSerpin1, and S. littoralis was also fed on transgenic Arabidopsis plants overproducing AtSerpin1. AtSerpin1 supplied in the artificial diet or by transgenic plants reduced the growth of $S$. littoralis larvae by $65 \%$ and $38 \%$, respectively, relative to controls. Nymphs of A. pisum exposed to diets containing AtSerpin 1 suffered high mortality levels $\left(\mathrm{LC}_{50}=637 \mu \mathrm{g} \mathrm{ml}^{-1}\right)$. The results indicate that AtSerpin 1 is a good candidate for exploitation in pest control.
\end{abstract}

Citation: Alvarez-Alfageme F, Maharramov J, Carrillo L, Vandenabeele S, Vercammen D, et al. (2011) Potential Use of a Serpin from Arabidopsis for Pest Control. PLoS ONE 6(5): e20278. doi:10.1371/journal.pone.0020278

Editor: Miguel A. Blazquez, Instituto de Biología Molecular y Celular de Plantas, Spain

Received January 27, 2011; Accepted April 25, 2011; Published May 31, 2011

Copyright: (C) 2011 Alvarez-Alfageme et al. This is an open-access article distributed under the terms of the Creative Commons Attribution License, which permits unrestricted use, distribution, and reproduction in any medium, provided the original author and source are credited.

Funding: The authors acknowledge the support of Ghent University, particularly the Industrial Research Fund (IOF) and the Multidisciplinary Research Partnership Ghent Bio-economy, of COST Action 862 to LC, and of Fund for Scientific Research-Flanders (FWO-Vlaanderen) to DV. SV was recipient of a BELSPO grant (12TKM106) and a Marie Curie Fellowship (MOIF-CT-2004). The funders had no role in study design, data collection and analysis, decision to publish, or preparation of the manuscript.

Competing Interests: The authors have declared that no competing interests exist.

*E-mail: fernando.alvarez@art.admin.ch (F-AA); guy.smagghe@ugent.be (GS)

wa Current address: Agroscope Reckenholz-Tänikon Research Station (ART), Zurich, Switzerland

ab Current address: Centro de Biotecnología y Genómica de Plantas (UPM-INIA), Universidad Politécnica de Madrid, Pozuelo de Alarcón, Madrid, Spain

ac Current address: Innogenetics N.V., Zwijnaarde, Belgium

ad Current address: Crop Design N.V., Zwijnaarde, Belgium

\section{Introduction}

Herbivorous pests of major crops are estimated to reduce yields by $8-15 \%$ worldwide [1]. Engineering crop plants for endogenous resistance to insect pests has been an important success of molecular technology. Currently, genetically modified (GM) plants expressing $\delta$-endotoxins from Bacillus thuringiensis $(B t)$ are providing significant control of agricultural insect pests and have reduced pesticide usage and production costs [2], [3]. The area sown with $B t$ crops has increased each year since 1996, when the first $B t$ crops were cultivated; in 2010, Bt crops were planted on 58 million hectares [4].

As farmers increasingly plant insect-resistant GM crops, selection pressure for the development of insect pests resistant to $B t$ toxins is also increasing. To date, field-evolved resistance has been documented in populations of five lepidopteran species [5]. Moreover, the efficacy of commercial $B t$ crops for some lepidopteran pests, such as the cotton leafworm Spodoptera littoralis Boisduval, is limited [6],[7], and phloem feeding pests including aphids are not susceptible to $B t$ crops [8]. Hence, complementary pest control strategies are necessary both to assure that the benefits provided by insect-resistant transgenic plants are not compromised and to target those pests that are not susceptible to $B t$ toxins. A summary of the strategies currently being investigated can be found in [8-11]. Among these, GM crops producing plant serine or cysteine protease inhibitors have been shown to confer resistance against a wide range of agricultural pests [12]. Protease inhibitors contribute to plant defense by inhibiting invertebrate proteases and, consequently, by reducing the availability of amino acids necessary for invertebrate growth and development. Transgenic plants expressing protease inhibitors, however, rarely achieve the same level of pest control as transgenic plants expressing $B t$ toxins [13] because herbivores are able to use several strategies to adapt to the inhibitors [12]. Still, plant protease inhibitors have the potential to be effective insecticidal proteins if insect adaptation to them can be overcome. For example, the combination of two protease inhibitors can lead to adverse effects on the target species that are not obtained with either inhibitor alone [14]. 
Serpins (serine protease inhibitors or classified inhibitor family I4) are the largest and most broadly distributed superfamily of protease inhibitors [15]. Serpin-like genes have been identified in animals, plants, bacteria, and some viruses [16]. Most serpins are irreversible inhibitors of serine proteases of the chymotrypsin family, although some have evolved to inhibit other types of serine proteases, and a few are also able to inhibit cysteine proteases [1721]. Furthermore, some serpins have the ability to form complexes with very divergent proteases [22]. Serpins are involved in a number of fundamental biological processes, and a role in the protection of storage tissue against insects and pathogens has been proposed for plant serpins [23], [24]. Consistent with the idea that serpins protect against plant pests, the survival and fecundity of the green peach aphid Myzus persicae (Sulz.) were strongly and negatively correlated with the level of the serpin CmPS-1 in the phloem sap of Cucurbita maxima Duchesne [25]. A related serpin from Cucurbita sativa L., CsPS-1, is also thought to play a role in defense against herbivores [26].

Here we assessed the potential of AtSerpin1, a serpin from Arabidopsis thaliana (L). Heynh., for pest control. In vitro assays were conducted to measure the inhibitory activity of AtSerpin l against a range of pest species that rely mainly on either serine or cysteine proteases for digestion. Because insect-resistant GM plants should ideally control target species without harming non-target arthropods, a decomposer, a pollinator, and a predator were included in these in vitro assays. Subsequently, two pest species, S. littoralis and the pea aphid Acyrthosiphon pisum (Harris), were used in in vivo assays on artificial diets containing AtSerpinl. Finally, transgenic Arabidopsis plants overproducing AtSerpinl were tested against $S$. littoralis.

\section{Materials and Methods}

\section{Invertebrates}

Pest species. A permanent colony of A. pisum was reared on broad bean, Vicia faba L., plants. A laboratory colony of $S$. littoralis was maintained on an agar-based artificial diet. The two-spotted spider mite, Tetranychus urticae Koch, was reared on Phaseolus vulgaris L. bean plants in the laboratory, and the Colorado potato beetle, Leptinotarsa decemlineata (Say), was reared on fresh leaves of Solanum tuberosum L. in the laboratory. Frozen larvae of the Mediterranean corn borer, Sesamia nonagrioides Lefèbvre, were provided by Dr. Félix Ortego (Centro de Investigaciones Biológicas, CSIC, Madrid, Spain). All stages of a permanent insect colony of the red flour beetle, Tribolium castaneum Herbst, and of the yellow mealworm, Tenebrio molitor L., were kept on wheat flour mixed with brewer's yeast $(10 / 1, w / w)$.

Non-target species. Large earth bumblebees, Bombus terrestris (L.), and green lacewings, Chrysoperla carnea (Stephens), were purchased from Biobest NV (Westerlo, Belgium) and reared in our laboratory for several generations with commercial sugar water and pollen and eggs of the flour moth Ephestia kuehniella Zeller, respectively. Common earthworms, Lumbricus terrestris L., were collected in an agricultural field in Ghent (Belgium) and frozen in the laboratory upon arrival.

All laboratory colonies were reared in environmental chambers at $24 \pm 2{ }^{\circ} \mathrm{C}, 65 \pm 5 \% \mathrm{RH}$, and a 16:8 h (L:D) photoperiod.

\section{Preparation of extracts}

Adults of $A$. pisum, T. castaneum, and T. molitor, and a mixture of all stages of $\mathcal{T}$. urticae were collected from the rearing colonies, homogenized in $0.15 \mathrm{M} \mathrm{NaCl}$, centrifuged at $10,000 \mathrm{~g}$ for $5 \mathrm{~min}$ and stored frozen at $-20^{\circ} \mathrm{C}$ until needed. Last instar larvae of $S$. littoralis, $S$. nonagrioides, $L$. decemlineata, and C. carnea, and adults of $L$. terrestris, B. terrestris, and C. carnea were dissected in ice-cold $0.15 \mathrm{M}$ $\mathrm{NaCl}$, and the midguts and contents were removed. Each midgut was subsequently homogenized in $500 \mu \mathrm{l}$ of $0.15 \mathrm{M} \mathrm{NaCl}$. The suspensions were then centrifuged at $10,000 \mathrm{~g}$ for $5 \mathrm{~min}$, and the supernatants were stored frozen at $-20^{\circ} \mathrm{C}$ until needed. Before extracts were frozen, total protein content was determined according to the method of Bradford [27] with bovine serum albumin (BSA) as a standard.

\section{Production of recombinant AtSerpin1}

Recombinant Atserpin 1 was produced and purified as described in Vercammen et al. [33]. The cDNA for the ORF of At $1 \mathrm{~g} 47710$ was obtained by RT-PCR with the following forward and reverse primers, provided with the adequate $5^{\prime}$ extensions by Gateway ${ }^{\circledR}$ cloning (Invitrogen, Merelbeke, Belgium): 5'-ATGGACGTGGGTGAATC-3' and 5'-TTAATGGAACGGATCAACAAC-3'. After recombination in pDEST17, the plasmid was introduced into E. coli strain BL21(DE3)pLysE, and production of the HIS6tagged protein was induced by incubation in $0.2 \mathrm{mM}$ isopropyl- $\beta$ D-thiogalactopyranoside for $24 \mathrm{~h}$. The protein was purified by metal ion affinity chromatography $\left(\mathrm{TALO}^{\mathrm{TM}} ; \mathrm{BD}\right.$, Franklin Lakes, NJ). Protein concentration and purity were checked by Bradford analysis (Bio-Rad, Nazareth, Belgium) and SDSpolyacrylamide gel electrophoresis (PAGE).

\section{In vitro inhibitory activity of AtSerpin 1 against invertebrate digestive proteases}

To elucidate the potential of AtSerpin 1 to inhibit invertebrate digestive proteases, several species known to rely either on serine or cysteine proteases for protein digestion were selected for in vitro experiments. Specifically, the ability of AtSerpin 1 to inhibit the trypsin- and chymotrypsin-like serine activities in extracts of $S$. littoralis, S. nonagrioides, T. molitor, L. terrestris, B. terrestris, and C. carnea was tested using the substrates ZPR-AMC $(\mathcal{N}$-carbobenzoxy-PheArg-7-amido-4-methylcoumarin) and SLLVT-AMC ( $\mathcal{N}$-Suc-LeuLeu-Val-Tyr-7-amido-4-methylcoumarin), respectively, and $0.1 \mathrm{M}$ Tris-HCl buffer (pH 9.0). Inhibition of the cathepsin B- and L-like cysteine activities by AtSerpin 1 in extracts of A. pisum, T. castaneum, L. decemlineata, and T. urticae was determined using the substrates ZRR-AMC ( $\mathcal{N}$-carbobenzoxy-Arg-Arg-7-amido-4-methylcoumarin) and ZPR-AMC, respectively, and $0.1 \mathrm{M}$ phosphate buffer ( $\mathrm{pH} 5.0$ ). The standard assay used $5 \mu \mathrm{g}$ of protein extract in a volume of $100 \mu \mathrm{l}$. AtSerpin 1 was added at different final concentrations, ranging from 0.15 to $10 \mu \mathrm{M}$, and was incubated with the extracts for $15 \mathrm{~min}$ at room temperature. The substrate was then added to a final concentration of $0.2 \mathrm{mM}$. The reaction was incubated at $30^{\circ} \mathrm{C}$ for $45 \mathrm{~min}$, and the emitted fluorescence was measured with a $365 \mathrm{~nm}$ excitation wavelength filter and a $465 \mathrm{~nm}$ emission wavelength filter. Results were expressed as a percentage of protease activity relative to that in the absence of the inhibitor. All assays were carried out in duplicate with pooled extracts.

\section{Generation of Arabidopsis plants overproducing AtSerpin 1}

Transgenic Arabidopsis plants overproducing AtSerpin1 were generated to further investigate the potential of the serpin against S. littoralis larvae. The cDNA for the ORF of Atg47710 was obtained by reverse transcription-PCR with the following forward and reverse primers, provided with the adequate $5^{\prime}$ extensions for Gateway ${ }^{\circledR}$ cloning: 5'-ATGGACGTGGGTGAATC-3' and 5'TTAATGGAACGGATCAACAAC-3'. The ORF was cloned into the binary vector $\mathrm{pB} 7 \mathrm{GW} 2$ [28] via Gateway ${ }^{\circledR}$ recombination. In the resulting vector, the ORF was under transcriptional 
control of the promoter of the cauliflower mosaic virus 35S (CaMV $35 \mathrm{~S})$; the glufosinate ammonium resistance gene was present to allow for transgene selection. Binary constructs were transformed into Agrobacterium tumefaciens strain C58C1RifR[pMP90], and transgenic Arabidopsis Columbia-0 were obtained via floral dip transformation [29] and subsequent selection. Serpin overexpression was assessed by immunoblotting using antisera against AtSerpin 1 [19]. Three single-locus homozygous lines with high transgenic protein expression were selected for further analysis by Western blot.

\section{In vivo effect of AtSerpin1}

In vivo experiments with $S$. littoralis and $A$. pisum were used to assess the potential of AtSerpinl for pest control. These two herbivorous species were selected because (i) they are serious pests of several agricultural crops, (ii) they rely on different proteolytical enzymes for protein digestion, and (iii) our in vitro studies demonstrated that they are both highly susceptible to AtSerpin 1 (see Results).

Effect of purified AtSerpin1 on $\boldsymbol{S}$. littoralis. Third-instar S. littoralis larvae $(8-10 \mathrm{mg}$ each) from the laboratory colony were starved for $4 \mathrm{~h}$ before the start of the bioassay. Subsequently, four larvae were placed in a Petri dish $(9 \mathrm{~cm}$ diameter) and fed ad libitum for 6 days with artificial diet containing 0 (control), 65, or $650 \mu \mathrm{g} \mathrm{g}^{-1}$ AtSerpin 1. Larvae were weighed on day 2, 4, and 6 . Each treatment was represented by 12 replicate Petri dishes.

At the end of the feeding assay, 24 larvae from the control and the $65 \mu \mathrm{g} \mathrm{g}^{-1}$ AtSerpinl treatment were selected randomly and the midguts were dissected. Susbsequently, the serine-like proteolytic activites trypsin, chymotrypsin, and elastase were quantified as described by Ortego et al. [30].

Effect of transgenic Arabidopsis overproducing AtSerpin1 on S. littoralis. Second-instar S. littoralis larvae $(2.5-3.0 \mathrm{mg}$ each) from the laboratory colony were starved for $4 \mathrm{~h}$ and transferred to pots planted with 4-week-old Arabidopsis: transgenic lines overproducing AtSerpinl (lines AtSerpin OE1, AtSerpin OE2, and AtSerpin ${ }^{\text {OE3 }}$ ) or the non-transformed line Col-0. Four larvae were confined per pot and allowed to feed for 4 days. Larvae were weighed on the second and the fourth day. Six pots per line were used, resulting in 24 larvae per treatment.

Both bioassays with $S$. littoralis were carried out in a growth chamber at $24 \pm 2{ }^{\circ} \mathrm{C}, \quad 65 \pm 5 \% \quad \mathrm{RH}$, and a $16: 8 \mathrm{~h} \quad(\mathrm{~L}: \mathrm{D})$ photoperiod.

Effect of purified AtSerpin1 on A. pisum survival. Reproductive adults from the $A$. pisum laboratory colony were collected and transferred to fresh bean leaves, where they were allowed to produce nymphs for $12 \mathrm{~h}$. Experimental arenas consisted of sachets containing $130 \mu \mathrm{l}$ of artificial diet as described by Shahnaz et al. [31]. Neonate $(<12 \mathrm{~h})$ A. pisum nymphs were then brushed carefully onto sachets containing AtSerpinl at concentrations ranging from 0 to $1000 \mu \mathrm{g} \mathrm{ml}^{-1}$. Fifteen nymphs were confined in each sachet, and three to six sachets were used per treatment. Nymphal survival was recorded after 3 days, and Abbott's correction for natural mortality was applied [32].

Effect of purified AtSerpin1 on A. pisum proteolytic activities. Neonate nymphs $(<12 \mathrm{~h})$ from the permanent $A$. pisum culture were placed on sachets containing 0 or $1000 \mu \mathrm{g} \mathrm{ml}^{-1}$ AtSerpin 1, and allowed to feed for $24 \mathrm{~h}$. Three sachets containing 15 nymphs were used per treatment. After the feeding period, aphids from every sachet were collected, homogenized in $0.15 \mathrm{M}$ $\mathrm{NaCl}$, and stored frozen at $-20^{\circ} \mathrm{C}$ until required. Finally, digestive enzyme activities were measured as described by Carrillo et al. [33].
Both bioassays with $A$. pisum were performed in a growth chamber at $24 \pm 2{ }^{\circ} \mathrm{C}, \quad 65 \pm 5 \% \quad \mathrm{RH}$, and a $16: 8 \mathrm{~h} \quad$ (L:D) photoperiod.

\section{Statistical analysis}

A one-way analysis of variance (ANOVA) followed by a Student-Newman-Keuls test was used to compare S. littoralis larval growth among the different treatments in both bioassays and to compare the proteolytic activities of $A$. pisum fed with artificial diet with or without AtSerpin 1. Proteolytic activities of $S$. littoralis larvae fed either with control diet or diet incorporating AtSerpin 1 were analyzed using the Mann-Whitney $U$ test because data were not normally distributed. Differences between treatments were considered significant at $P<0.05$. The concentration of AtSerpin 1 causing $50 \%$ mortality $\left(\mathrm{LC}_{50}\right)$ on aphid nymphs was analyzed using nonlinear sigmoid curve fitting using the GraphPad Prism 4.0 software (GraphPad Software Inc., La Jolla, CA).

\section{Results}

\section{In vitro inhibitory activity of AtSerpin 1 against invertebrate digestive proteases}

The inhibitory activity of AtSerpin l was tested in vitro against serine or cysteine proteases from several invertebrate pest and non-target species (Table 1). The inhibition of trypsin- and chymotrypsin-like serine activities was investigated in extracts of the pests $S$. littoralis, $S$. nonagrioides, and T. molitor, and in extracts of the non-targets L. terrestris, B. terrestris, and C. carnea (Table 2). For all species, inhibition of trypsin activity by $10 \mu \mathrm{M}$ AtSerpin 1 was higher than $80 \%$. The trypsin activities of the non-targets $C$. carnea larvae and $B$. terrestris were highly susceptible to AtSerpin 1 , with an inhibition of $70 \%$ and $90 \%$, respectively, at the lowest concentration tested $(0.15 \mu \mathrm{M})$. AtSerpinl also inhibited chymotrypsin activity in all species tested, except in the case of $S$. nonagrioides.

The inhibition of cathepsin B- and L-like cysteine activities was determined in extracts of the pest species $A$. pisum, T. castaneum, $L$. decemlineata, and $T$. urticae (Table 2). AtSerpinl inhibited the hydrolysis of the substrate ZRR-AMC in all species studied, although it never caused more than $75 \%$ inhibition, suggesting that cathepsin B activity is much less susceptible than trypsin activity to AtSerpin 1. Inhibition of cathepsin L activity was also detected in these four species.

\section{Effect of AtSerpin1 on S. littoralis}

Bioassay with artificial diet. Ingestion of artificial diets containing the protease inhibitor AtSerpin 1 markedly reduced the weight gain of $S$. littoralis (Figure 1). A significant difference $(P<0.001)$ occurred after only 2 days of exposure when third instars were reared on artificial diet containing $650 \mu \mathrm{g} \mathrm{g}^{-1}$ AtSerpin1. This difference continued throughout the bioassay, and on day 6 , the weight increase was $65 \%(P<0.001)$ lower for $S$. littoralis larvae ingesting the inhibitor than for the control. For larvae exposed to $65 \mathrm{\mu g} \mathrm{g}^{-1}$ AtSerpinl, weight gain was significantly reduced by $20 \%$ on day 4 and by $33 \%$ on day 6 relative to the control (Figure 2).

To investigate the physiological background, biochemical analysis were carried out on guts of $S$. littoralis larvae dissected at the end of the feeding assay. Trypsin and chymotrypsin activities were significantly reduced in those fed on artificial diet incorporating $65 \mathrm{\mu g} \mathrm{g}^{-1}$ AtSerpin 1 compared to those feeding on control diet, whereas no differences were observed for elastase activity (Figure 2).

Bioassay with transgenic Arabidopsis. Three transgenic Arabidopsis lines overproducing AtSerpinl were tested against $S$. 
Table 1. Ecological function and main digestive proteases of the invertebrate species tested in vitro against AtSerpin1.

\begin{tabular}{llll}
\hline & & & \\
\hline Species name & Ecological function & Main proteases & Reference \\
\hline Spodoptera littoralis (Lepidoptera: Noctuidae) & Herbivory & SEP & {$[34]$} \\
Sesamia nonagrioides (Lepidoptera: Noctuidae) & Herbivory & SEP & {$[30]$} \\
Tenebrio molitor (Coleoptera: Tenebrionidae) & Herbivory & SEP, CEP* & {$[35]$} \\
Lumbricus terrestris (Annelida: Lumbricidae) & Decomposition & SEP & {$[36]$} \\
Bombus terrestris (Hymenoptera: Apidae) & Pollination & SEP & {$[37]$} \\
Chrysoperla carnea (Neuroptera: Chrysopidae) & Predation & SEP & {$[38]$} \\
Acyrthosiphon pisum (Homoptera: Aphididae) & Herbivory & CEP & 33 \\
Tribolium castaneum (Coleoptera: Tenebrionidae) & Herbivory & CEP & {$[39]$} \\
Leptinotarsa decemlineata (Coleoptera: Chrysomelidae) & Herbivory & CEP & {$[40]$} \\
Tetranychus urticae (Acari: Tetranychidae) & Herbivory & CEP & {$[41]$} \\
\hline Abbrevi & & \\
\hline
\end{tabular}

Abbreviations: SEP = serine endoproteases; $C E P=$ cysteine endoproteases.

*Only SEP were tested against AtSerpin1.

doi:10.1371/journal.pone.0020278.t001

littoralis Expression of AtSerpinl in leaves of Arabidopsis was confirmed by Western blot using increasing concentrations of purified AtSerpinl (Figure 3). Differences in the AtSerpinl expression among the transgenic lines was observed, being higher in AtSerpin ${ }^{\text {OE2 }}$ and AtSerpin ${ }^{\text {OE3 }}$. In those lines, about $5 \mathrm{ng}$ AtSerpin ug ${ }^{-1}$ of total protein content was measured.

Second-instar larvae were fed for 4 days on transgenic or nontransformed plants, and the increase of weight was measured (Figure 4). No significant differences were observed when the transgenic lines AtSerpin ${ }^{\mathrm{OE} 1}$ and AtSerpin ${ }^{\mathrm{OE} 2}$ were compared with the control plants. However, the increase of weight was $25 \%$ lower $(P<0.001)$ on day 2 and $38 \%$ lower $(P<0.001)$ on day 4 for $S$. littoralis larvae reared on the transgenic AtSerpin ${ }^{\text {OE3 }}$ than for larvae fed on non-transformed plants.

Effect of AtSerpin1 on $A$. pisum

A. pisum nymphs reared for 3 days on diets containing 100 to $1000 \mu \mathrm{g} \mathrm{ml}^{-1}$ AtSerpinl were highly susceptible to the inhibitor (Figure 5). Mortality reached $77.4 \%$ when A. pisum were fed $1000 \mu \mathrm{g} \mathrm{ml}^{-1}$ of the serpin. The effective AtSerpin1 concentra-

Table 2. In vitro inhibitory activity of the protease inhibitor AtSerpin 1 against trypsin- and chymotrypsin-like serine, and cathepsin B- and cathepsin L-like cysteine activities in extracts of several pest and non-target invertebrate species.

\begin{tabular}{|c|c|c|c|c|c|c|}
\hline \multirow[b]{3}{*}{ Species name } & \multicolumn{6}{|c|}{ Inhibition (\%) } \\
\hline & \multicolumn{3}{|c|}{ Trypsin activity } & \multicolumn{3}{|c|}{ Chymotrypsin activity } \\
\hline & $0.15 \mu M$ & $1.25 \mu \mathrm{M}$ & $10 \mu \mathrm{M}$ & $0.15 \mu \mathrm{M}$ & $1.25 \mu \mathrm{M}$ & $10 \mu \mathrm{M}$ \\
\hline Spodoptera littoralis* & $11.8 \pm 0.8$ & $52.3 \pm 0.3$ & $90.4 \pm 0.7$ & $18.2 \pm 4.0$ & $30.7 \pm 15.3$ & $39.7 \pm 14.8$ \\
\hline Sesamia nonagrioides* & $36.0 \pm 1.3$ & $89.8 \pm 1.3$ & $97.5 \pm 0.3$ & $\mathrm{ni}$ & $\mathrm{ni}$ & $\mathrm{ni}$ \\
\hline Tenebrio molitor* & $51.2 \pm 3.5$ & $94.9 \pm 0.6$ & $92.3 \pm 3.6$ & $7.2 \pm 0.8$ & $14.1 \pm 2.3$ & $45.3 \pm 3.8$ \\
\hline Lumbricus terrestris $^{\dagger}$ & $\mathrm{ni}$ & ni & $82.7 \pm 1.2$ & - & - & - \\
\hline Bombus terrestris $^{\dagger}$ & $91.8 \pm 0.5$ & $97.8 \pm 0.3$ & $98.5 \pm 0.1$ & - & - & - \\
\hline \multicolumn{7}{|l|}{ Chrysoperla carnea ${ }^{\dagger}$} \\
\hline Larvae & $66.6 \pm 3.5$ & $94.0 \pm 3.2$ & $98.8 \pm 1.3$ & $\mathrm{ni}$ & $51.1 \pm 7.2$ & $75.5 \pm 1.3$ \\
\hline \multirow[t]{3}{*}{ Adults } & $56.2 \pm 1.5$ & $99.2 \pm 0.4$ & $99.5 \pm 1.0$ & ni & $15.8 \pm 2.5$ & $66.1 \pm 5.7$ \\
\hline & \multicolumn{3}{|c|}{ Cathepsin B activity } & \multicolumn{3}{|c|}{ Cathepsin L activity } \\
\hline & $0.15 \mu \mathrm{M}$ & $1.25 \mu \mathrm{M}$ & $10 \mu \mathrm{M}$ & $0.15 \mu \mathrm{M}$ & $1.25 \mu \mathrm{M}$ & $10 \mu \mathrm{M}$ \\
\hline Acyrthosiphon pisum* & $38.6 \pm 1.3$ & $49.9 \pm 0.1$ & $55.5 \pm 1.7$ & $38.3 \pm 2.2$ & $42.4 \pm 2.7$ & $39.2 \pm 4.3$ \\
\hline Tribolium castaneum* & $\mathrm{ni}$ & ni & $75.1 \pm 3.6$ & $\mathrm{ni}$ & $\mathrm{ni}$ & $69.8 \pm 3.5$ \\
\hline Leptinotarsa decemlineata* & $13.5 \pm 13.4$ & $40.6 \pm 8.5$ & $45.8 \pm 10.6$ & $\mathrm{ni}$ & $3.9 \pm 9.9$ & $47.8 \pm 3.8$ \\
\hline Tetranychus urticae* & $\mathrm{ni}$ & $\mathrm{ni}$ & $46.7 \pm 25.0$ & $\mathrm{ni}$ & $\mathrm{ni}$ & $24.6 \pm 3.1$ \\
\hline
\end{tabular}

The percentage of inhibition was calculated as [(1 - activity with AtSerpin1/activity without AtSerpin 1$) \times 100]$. Values represent mean+SE for duplicated independent determinations from a unique pool of extracts.

*pest species;

'non-target species.

"ni" denotes no inhibition.

doi:10.1371/journal.pone.0020278.t002 


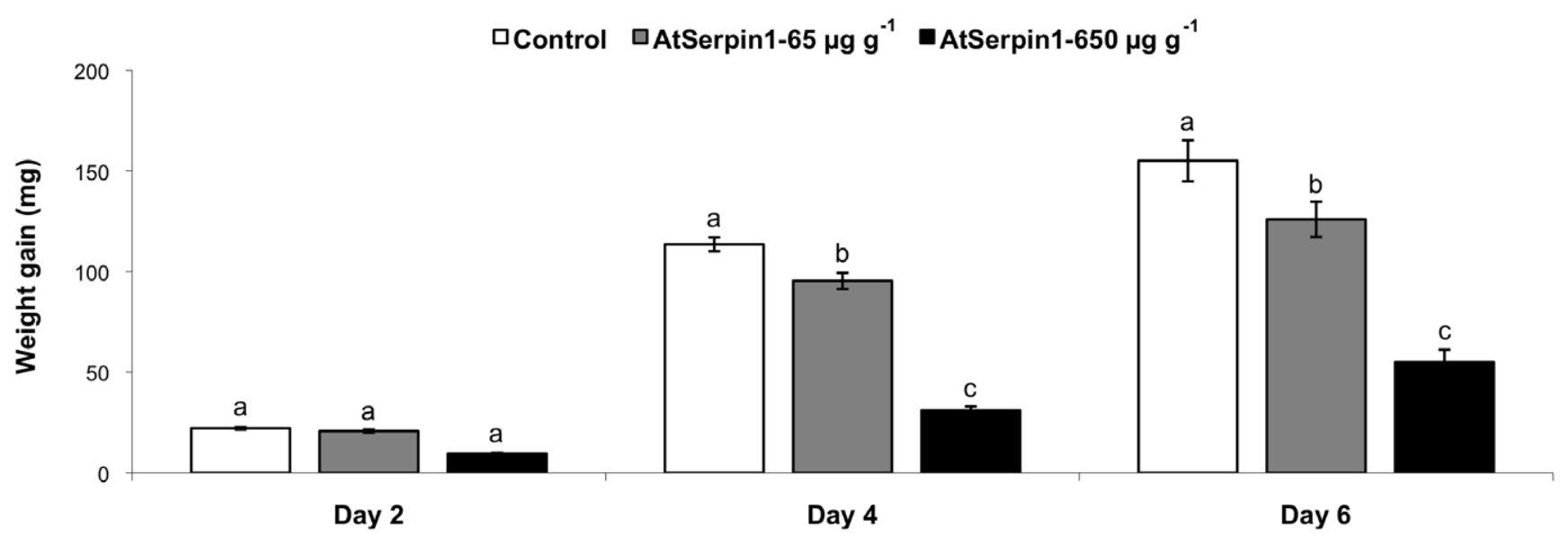

Figure 1. Weight gain of Spodoptera littoralis larvae fed on a diet containing 65 or $650 \mu \mathrm{g} \mathrm{g}^{-1}$ AtSerpin1 or control diet without inhibitor. Feeding assays were performed for 6 days with third-instar larvae. Bars represent mean \pm SE. Bars with different letters on the same day are significantly different $(P<0.05$; one-way ANOVA followed by Student-Newman-Keuls) $(N=48)$. doi:10.1371/journal.pone.0020278.g001

tion for $50 \%$ mortality $\left(\mathrm{LC}_{50}\right)$ at the third day of feeding was $637 \mu \mathrm{g} \mathrm{ml}^{-1} \quad\left(95 \% \quad\right.$ confidence limits $\left.=367-1105 ; \quad R^{2}=0.91\right)$ (Figure 5). Hence, it appears that AtSerpinl not only inhibits cysteine proteases in A. pisum extracts in vitro but also has a strong insecticidal effect on nymphs.

To investigate the response of proteolytical enzymes of $A$. pisum to the ingestion of AtSerpin1, nymphs were fed with a diet containing $1000 \mu \mathrm{g} \mathrm{ml}^{-1}$ AtSerpinl or a control diet without the inhibitor for $24 \mathrm{~h}$, and proteolytic activities were subsequently quantified (Table 3). The cathepsin B- and L-like cysteine activities were significantly reduced (by $37 \%$ and $47 \%$, respectively) in nymphs fed with AtSerpin 1 . In contrast, leucine aminopeptidase activity was enhanced by $42 \%$ when aphids were exposed to the inhibitor. Lastly, no differences were observed in carboxypeptidase $\mathrm{A}$ and $\mathrm{B}$ activities in $A$. pisum nymphs that were fed a diet with or without AtSerpinl.

\section{Discussion}

Although many plant protease inhibitors from the serpin superfamily have been identified and hypothesized to have a role in host defense, to our knowledge only Yoo et al. [25] and the current study have investigated the potential of a serpin for pest control.

In vitro inhibitory activity of AtSerpin 1 against invertebrate digestive proteases

In vitro studies revealed that AtSerpin l has a broad spectrum of activity because it inhibited both serine and cysteine proteases from a wide range of organisms, including the common earthworm ( $L$. terrestris), the two-spotted spider mite ( $T$. urticae), and eight insect species belonging to five different orders. Two

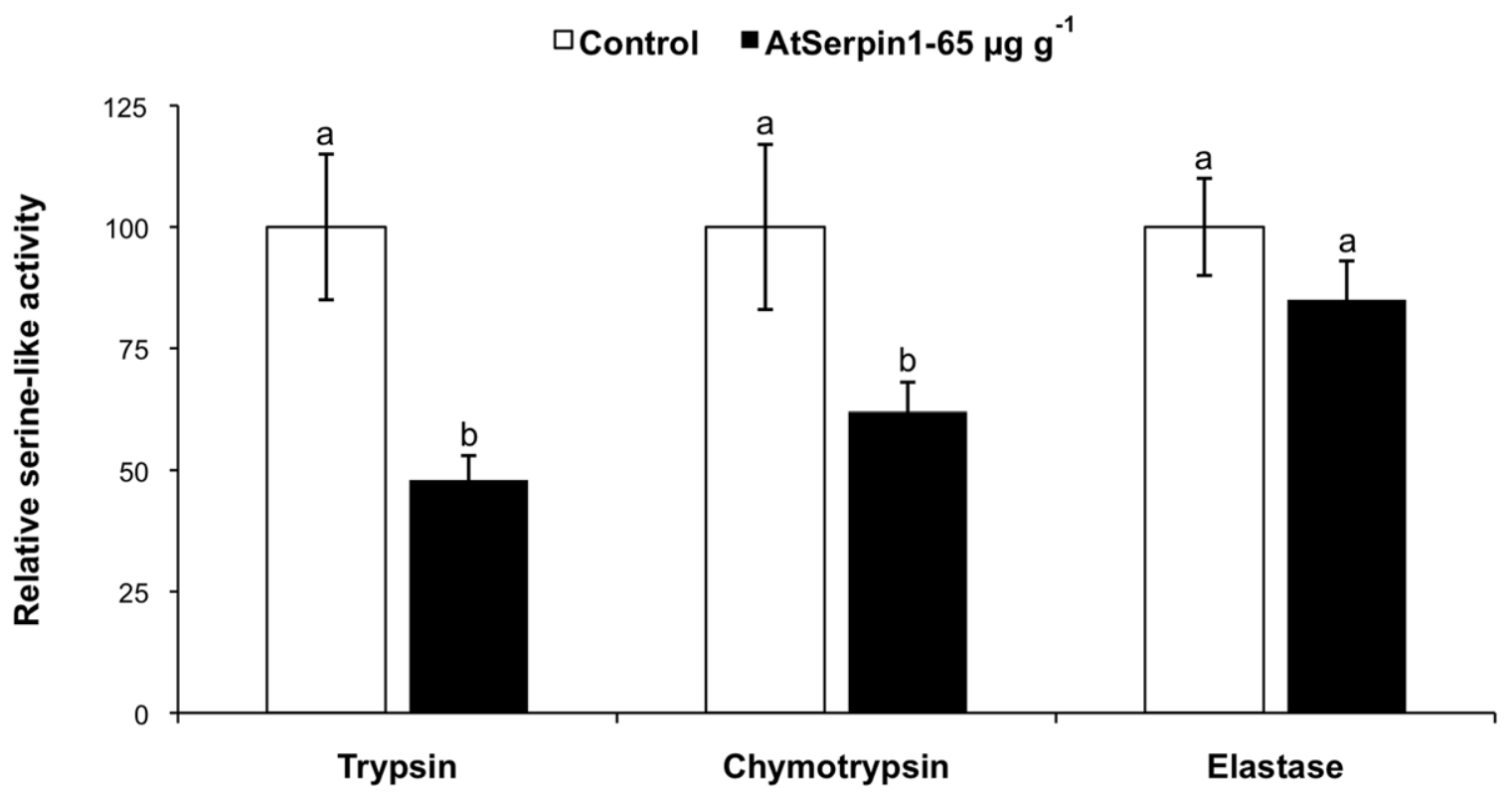

Figure 2. Serine-like proteolytic activities of Spodoptera littoralis third-instar larvae fed for 6 days on a diet containin $65 \mu \mathrm{g} \mathrm{g}^{-1}$ AtSerpin 1 or control diet without inhibitor. Bars represent mean \pm SE. Bars with different letters are significantly different $(P<0.05 ;$ MannWhitney $U$ test) $(N=24)$.

doi:10.1371/journal.pone.0020278.g002 


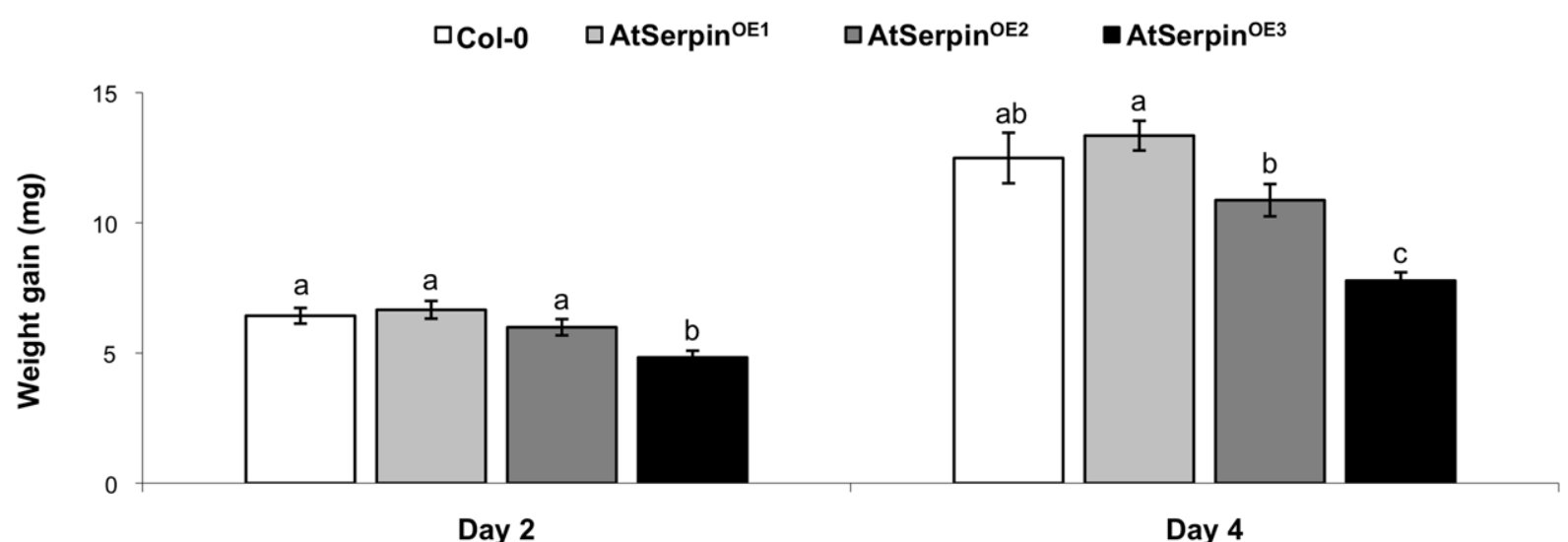

Figure 3. Western blot immunoassay showing the expression of AtSerpin1 in leaves of the transgenic Arabidopsis lines AtSerpin $1{ }^{\text {OE1 }}$, AtSerpin $1{ }^{\text {OE2 }}$, and AtSerpin $1{ }^{\text {OE3 }}$, and the non-transformed line Col-0. Lanes: (1) Page ruler plus protein standard; (2) 100 ng AtSerpin1; (3) $50 \mathrm{ng} \mathrm{AtSerpin1;} \mathrm{(4)} 25 \mathrm{ng}$ AtSerpin1; (5) $12.5 \mathrm{ng}$ AtSerpin1; (6) $5 \mathrm{ng}$ AtSerpin1; (7) $0 \mathrm{ng}$ AtSerpin1; (8) overproducing line AtSerpin OE3 $(6 \mathrm{ng})$; (9) overproducing line AtSerpin ${ }^{\mathrm{OE} 2}(6 \mathrm{ng})$; (10) overproducing line AtSerpin ${ }^{\mathrm{OE} 1}$ (6 ng); (11) non-transformed line Col-0. In lanes 7-9, the upper band is the full-length and active form of AtSerpin1, while the lower band is the cleaved form after interaction with a protease. doi:10.1371/journal.pone.0020278.g003

recent studies have demonstrated the ability of AtSerpin1 to inhibit cysteine proteases [19], [42]; the potential of AtSerpin l to target serine proteases, however, has never been reported before. Although most serpins inhibit either serine or cysteine proteases [16], some can inhibit proteases from several families. For example, the mouse serpin SON-5 is a dual inhibitor of both chymotrypsin-like serine and the papain-like cysteine proteases [43]. Brüning et al. [44] showed that a serpin, Spn4, from the fruit fly Drosophila melanogaster Meigen inhibits proteases from three different families.

The role of plant serpins in the protection of crops against insects has been proposed [23], [24], but very little is known about the potential of such protease inhibitors to control agricultural pests. To address this question, we selected two species, $S$. littoralis and $A$. pisum, for further in vivo studies (discussed in the next two sections). We selected these species in part because $S$. littoralis relies mainly on serine proteases, $A$. pisum relies mainly on cysteine proteases, and both were susceptible to AtSerpinl in the in vitro experiments.

\section{In vivo effect of AtSerpin1 on S. littoralis}

Serine proteases provide the major midgut endoproteolytic activities in S. littoralis larvae [34], and previous studies have demonstrated that transgenic plants expressing serine protease inhibitors can confer resistance against $S$. littoralis [45], [46]. When the protease inhibitor AtSerpin 1 was incorporated into an artificial

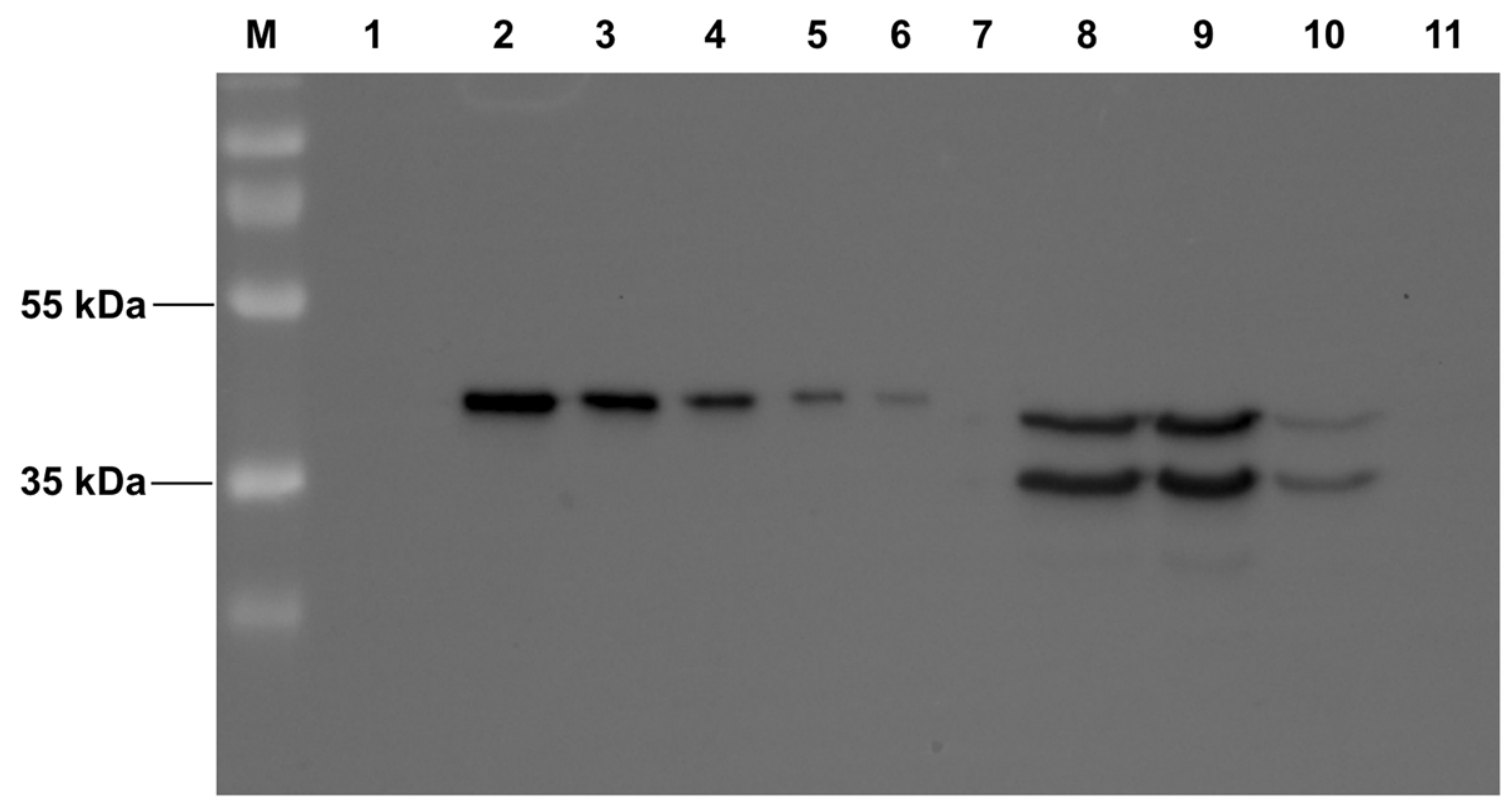

Figure 4. Weight gain of Spodoptera littoralis larvae fed on transgenic Arabidopsis plants overproducing AtSerpin 1 (lines AtSerpin $^{\mathrm{OE} 1}$, AtSerpin ${ }^{\mathrm{OE} 2}$, and AtSerpin ${ }^{\mathrm{OE} 3}$ ) or on non-transformed plants (line Col-0). Feeding assays were performed for 4 days with second-instar larvae. Bars represent mean \pm SE. Bars with different letters on the same day are significantly different $(P<0.05 ;$ one-way ANOVA followed by Student-Newman-Keuls) ( $N=24)$.

doi:10.1371/journal.pone.0020278.g004 


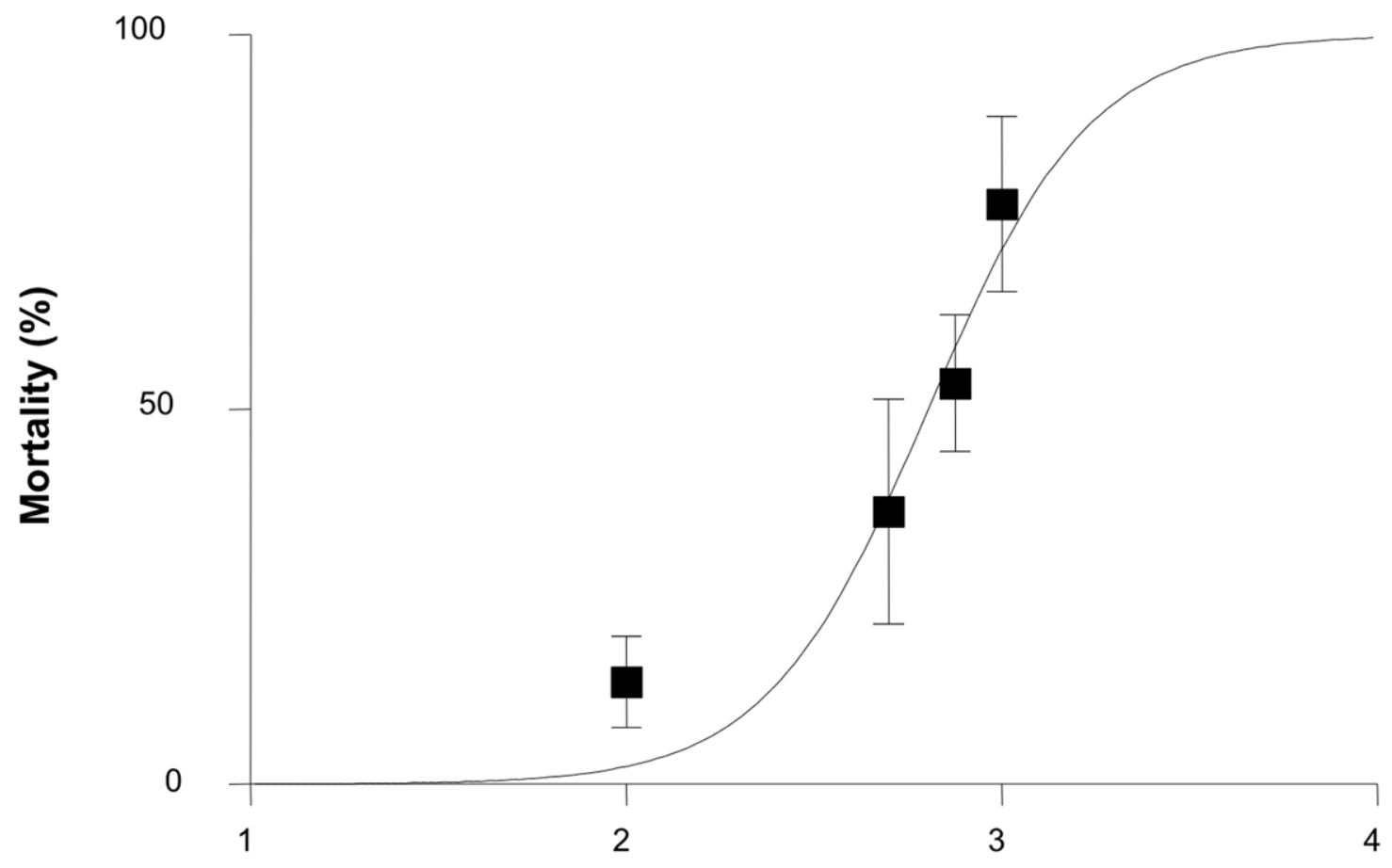

\section{Log AtSerpin1 $\left(\mu \mathrm{g} \mathrm{ml}^{-1}\right)$}

Figure 5. Concentration-response curve for mortality of newborn Acyrthosiphon pisum nymphs fed for 3 days with artificial diet containing increasing concentrations of the protease inhibitor AtSerpin1. Points represent mean \pm SE. Three to six replicates with 15 nymphs each were used per concentration.

doi:10.1371/journal.pone.0020278.g005

diet, the weight gain of $S$. littoralis larvae was substantially reduced relative to the control. The observed effects of AtSerpin 1 on larval weight gain were correlated with a significant decreased of midgut trypsin activity. Weight gain also was reduced when $S$. littoralis were fed with transgenic Arabidopsis plants overproducing the serpin.

Table 3. Proteolytic activities of Acyrthosiphon pisum adults after 1 day of feeding on a control diet (AtSerpin 1-) or a diet containing $1000 \mu \mathrm{g} \mathrm{ml}^{-1}$ AtSerpin1 (AtSerpin1+).

\begin{tabular}{|c|c|c|c|}
\hline \multirow[b]{2}{*}{ Protease } & \multirow[b]{2}{*}{ pH } & \multicolumn{2}{|c|}{ Specific activity $^{a}$} \\
\hline & & AtSerpin 1- & AtSerpin1+ \\
\hline \multicolumn{4}{|l|}{ Cysteine protease } \\
\hline Cathepsin B & 6.5 & $4.0 \pm 0.36^{\mathrm{a}}$ & $2.5 \pm 0.19^{b}$ \\
\hline Cathepsin L & 3 & $18.3 \pm 0.87^{a}$ & $9.6 \pm 0.34^{b}$ \\
\hline Cathepsin L & 5.5 & $13.9 \pm 1.43^{\mathrm{a}}$ & $7.8 \pm 0.47^{b}$ \\
\hline Leucine amino peptidase & 7 & $8.7 \pm 0.93^{\mathrm{a}}$ & $12.4 \pm 0.84^{\mathrm{b}}$ \\
\hline Carboxypeptidase A & 7 & $9.2 \pm 0.50^{\mathrm{a}}$ & $8.4 \pm 0.40^{\mathrm{a}}$ \\
\hline Carboxypeptidase B & 8 & $12.6 \pm 0.44^{\mathrm{a}}$ & $12.3 \pm 0.90^{\mathrm{a}}$ \\
\hline
\end{tabular}

${ }^{\text {aSpecific activities as nmoles of substrate hydrolyzed } \mathrm{min}^{-1} \mathrm{mg} \text { protein }}{ }^{-1}$.

Values are mean \pm SE of triplicate measurements from three independent replicates.

Means followed by the same letter within a row are not significantly different from each other $(P \leq 0.05$; one-way ANOVA followed by Student-NewmanKeuls).

doi:10.1371/journal.pone.0020278.t003
The results obtained in our bioassays are in agreement with many studies that have shown the potential of different plant serine protease inhibitors to interfere with the performance of lepidopteran species, either when the inhibitors are incorporated into an artificial diet or when they are expressed in transgenic plants [8], [12], [47]. However, the level of pest control that is routinely provided by $B t$ toxins is rarely provided by serine protease inhibitors, including AtSerpin1. It is well known that lepidopteran pests possess a remarkable ability to adapt their digestive proteolytic metabolism to the dietary material ingested and, therefore, to counteract the inhibitory activity of protease inhibitors [48], [49]. For this reason, researchers have suggested that a combination of two or more inhibitors may be required to overcome the capacity of such species to adapt to protease inhibitors. For example, Dunse et al. [14] recently demonstrated that growth of larvae of the cotton bollworm Helicoverpa armigera (Hübner) was substantially decreased on an artificial diet containing two serine protease inhibitors but not on a diet containing one serine protease inhibitor.

\section{In vivo effect of AtSerpin1 on $A$. pisum}

Cysteine proteases have been identified in several aphid species [50], [51], including A. pisum [33], [52], [53]. Our in vitro assays showed that AtSerpinl strongly inhibits cathepsin B and L protease activities of whole A. pisum extracts, and when administered into an artificial diet, AtSerpinl was toxic to $A$. pisum nymphs with $50 \%$ mortality at $637 \mu \mathrm{g} \mathrm{ml}{ }^{-1}$. Researchers previously suggested that a serpin from C. maxima (CmPS-1) plays a role in plant defence against aphids; feeding assays established a correlation between increase in CmPS-1 within the phloem sap 
and the reduced ability of $M$. persicae to survive and reproduce on C. maxima plants [25]. However, survival of neonate $M$. persicae nymphs fed on a sucrose solution supplemented with $200 \mu \mathrm{g} \mathrm{ml}$ of purified CmPS-1 was not reduced. This might be because CmPS-1 requires additional phloem proteins to form an active complex [25].

Some studies have reported deleterious effects of plant cysteine protease inhibitors on aphids fed on artificial diets. The cystatin OC-I induced moderate but significant growth inhibition on three aphid species: A. pisum, the cotton aphid Aphis gossypii Glover, and M. persicae [54]. Likewise, diets supplemented with OC-I (ranging from 20 to $500 \mu \mathrm{g} \mathrm{ml}^{-1}$ ) significantly reduced nymphal survival of the potato aphid Macrosiphum euphorbiae (Thomas) and prevented aphids from reproducing [55]. Artificial diets containing either a modified version of OC-I or the recombinant chicken egg white cystatin $(\mathrm{CEW})$ reduced the survival and growth of $M$. persicae nymphs [56]. The barley cystatin HvCPI-6 was toxic to A. pisum nymphs $\left(\mathrm{LC}_{50}=150 \mu \mathrm{g} \mathrm{ml}^{-1}\right)$ [33]. Moreover, the developmental time of $A$. pisum was significantly delayed when newborn nymphs were fed for 1 day on diet containing HvCPI-6 at $400 \mu \mathrm{g} \mathrm{ml}^{-1}$ and were subsequently placed on bean plants until they reached adulthood [33].

In the current study, the effect of AtSerpinl on nymphal mortality was correlated with a significant decrease of cathepsin B and $\mathrm{L}$ protease activities after the nymphs fed on artificial diet containing serpin. In addition, leucine aminopeptidase activity was enhanced, suggesting a compensatory response to the inhibitory effect mediated by AtSerpin1. The overproduction of non-target proteases as a response to plant defense proteins is common in herbivorous arthropods [48], [57]. In a bioassay similar to the one described here, the ingestion of HvCPI- 6 by $A$. pisum and $M$. persicae nymphs was correlated with a decrease of cathepsin B and $\mathrm{L}$ protease activities, and in the case of $M$. persicae, an increase of leucine aminopeptidase activity [33]. Because the artificial diet used in both studies was protein free, the results suggest that the toxicity of the serpin was not linked to disruption of food protein digestion but to the disruption of non-digestive proteases involved in other physiological processes. The cysteine protease inhibitor from rice, oryzacystatin (OC-I), not only affected the aphid $M$. persicae through digestive tract targets but also inhibited extradigestive proteolytic activities in the hemolymph and internal organs [54]. Similar to our findings, the effects of OC-I on $M$. persicae were correlated with a reduction of a major cysteine-like protease activity in whole adult extracts [54].

\section{References}

1. Oerke EC (2006) Crop losses to pests. J Agri Sci 144: 31-43.

2. Toenniessen GH, O'Toole JC, DeVries J (2003) Advances in plant biotechnology and its adoption in developing countries. Curr Opin Plant Biol 6: 191-198.

3. Brookes G, Barfoot P (2005) GM crops: the global economic and environmental impact: the first nine years 1996-2004. Ag Bio Forum 8: 15

4. James C (2010) Global status of commercialized biotech/GM Crops: 2010. ISAAA Brief No. 42, International Service for the Acquisition of Agri-Biotech Applications, Ithaca, NY, USA.

5. Carrière Y, Crowder DW, Tabashnik BE (2010) Evolutionary ecology of insect adaptation to Bt crops. Evolut Appl 3: 561-573.

6. Pilcher CD, Rice MD, Obrycki JJ, Lewis LC (1997) Field and laboratory evaluation of transgenic Bacillus thuringiensis corn on secondary Lepidopteran pests (Lepidoptera: Noctuidae). J Econ Entomol 90: 669-678.

7. Dutton A, Romeis J, Bigler F (2005) Effects of Bt maize expressing CrylAb and Bt spray on Spodoptera littoralis. Entomol Exp Appl 114: 161-169.

8. Malone LA, Gatehouse AMR, Barratt BIP (2008) Beyond Bt: Alternative strategies for insect-resistant genetically modified crops. In: Integration of insectresistant genetically modified crops within IPM programs J. Romeis, AM. Shelton, GG. Kennedy, eds. Springer Science+Business Media B.V.: pp 357-417.

9. Ferry N, Edwards MG, Gatehouse JA, Gatehouse AMR (2004) Plant-insect interactions: molecular approaches to insect resistance. Curr Opin Biotech 15: $155-161$.

\section{Concluding remarks}

Before commercial release, GM crops must undergo an environmental risk assessment to ensure that they do not cause unacceptable detrimental effects to non-target organisms. This is especially relevant in the case of plants producing protease inhibitors, given that these inhibitors may affect many different organisms. Our in vitro assays showed that the serine proteases of the three non-target species tested were highly inhibited by AtSerpin 1. Therefore, if GM plants producing AtSerpin 1 are to be deployed in the future, the impact on non-target organisms should be taken into account and special attention should be given to the routes of exposure.

In vivo assays with $S$. littoralis and $A$. pisum showed very promising results for pest control by AtSerpin1. Artificial diet and plant bioassays have demonstrated that AtSerpinl reduces the growth of S. littoralis larvae but does not cause mortality. For AtSerpin 1 to make a meaningful contribution to plant resistance against $S$. littoralis, the efficacy of the serpin must be increased either by protein engineering [12] or by using it in combination with other protease inhibitors (see above) or with other pesticidal proteins. Interestingly, A. pisum nymphs incurred high mortality levels when exposed to AtSerpinl through artificial diet. Some studies have previously shown that transgenic plants producing cysteine protease inhibitors can confer partial resistance against aphid species [33], [54], [58]. Future experiments with A. pisum should therefore determine whether the detrimental effect observed with an artificial diet bioassay in the current study is obtained with AtSerpin1-expressing transgenic plants.

\section{Acknowledgments}

We are grateful to Félix Ortego and Olivier Sanvido for their valuable comments on earlier versions of this manuscript. We also thank Didier Van de Velde for his technical assistance and Jurgen Haustraete (Protein Service Facility, Department for Molecular Biomedical Research, VIB, Ghent, Belgium) for assistance in protein purification methodology.

\section{Author Contributions}

Conceived and designed the experiments: FAA JM LC SV DV FVB GS. Performed the experiments: FAA JM LG SV DV. Analyzed the data: FAA JM LC GS. Contributed reagents/materials/analysis tools: FVB GS. Wrote the paper: FAA LC FVB GS.

10. Christou P, Capell T, Kohli A, Gatehouse JA, Gatehouse AMR (2006) Recent developments and future prospects in insect pest control in transgenic crops. Trends Plant Sci 11: 302-308.

11. Gatehouse JA (2008) Biotechnological prospects for engineering insect-resistant plants. Plant Physiol 146: 881-887.

12. Schlüter U, Benchabane M, Munger A, Kiggundu A, Vorster J, et al. (2010) recombinant protease inhibitors for hervibore pest control: a multitrophic perspective. J Exp Bot 61: 4169-4183.

13. Ferry N, Edwards MG, Gatehouse JA, Capell T, Christou P, et al. (2006) Transgenic plants for insect pest control: a forward looking scientific perspective. Transgenic Res 15: 13-19.

14. Dunse KM, Stevens JA, Lay FT, Gaspar YM, Heath RL, et al. (2010) Coexpression of potato type I and II proteinase inhibitors gives cotton plants protection against insect damage in the field. Proc Natl Acad Sci USA 107: 15011-15015.

15. Rawlings ND, Tolle DP, Barrett AJ (2004) MEROPS: the peptidase database. Nucleic Acids Res 32 Database issue: D160-D164.

16. Gettins PGW (2002) Serpin structure, mechanism, and function. Chem Rev 102: 4751-4803.

17. Schick C, Brömme D, Bartuski A, Uemura Y, Schechter N, et al. (1998) The reactive site loop of the serpin SCCAl is essential for cysteine proteinase inhibition. Proc Natl Acad Sci USA 95: 13465-70. 
18. McGowan S, Buckle A, Irving J, Ong P, Bashtannyk-Puhalovich T, et al. (2006) $\mathrm{X}$-ray crystal structure of MENT: evidence for functional loop-sheet polymers in chromatin condensation. EMBO J 25: 3144-55.

19. Vercammen D, Belenghi B, van de Cotte B, Beunens T, Gavigan JA, et al. (2006) Serpin 1 of Arabidopsis thaliana is a suicide inhibitor for metacaspase 9. J Mol Biol 364: 625-636.

20. Ong PG, McGowan S, Pearce MC, Irving JA, Kan WT, et al. (2007) DNA accelerates the inhibition of human cathepsin V by serpins. J Biol Chem 282: 36980-36986.

21. Roberts TH, Hejgaard J (2008) Serpins in plants and green algae. Funct Integr Genomics 8: 1-27.

22. Huntington JA (2006) Shape-shifting serpins- advantages of a mobile mechanism. TiBS 31: 427-435.

23. Dahl SW, Rasmussen SK, Hejgaard J (1996) Heterologous expression of three plant serpins with distinct inhibitory specificities. J Biol Chem 271: 25083-25088.

24. Rasmussen SK, Dahl SW, Norgard A, Hejgaard J (1996) A recombinant wheat serpin with inhibitory activity. Plant Mol Biol 30: 673-677.

25. Yoo B-C, Aoki K, Campbell LR, Hull RJ, Xoconostle-Cazares B, et al. (2000) Characterization of Cucurbita maxima Phloem Serpin-1 (CmPS-1). J Biol Chem 275: $35122-35128$

26. Kehr J (2006) Phloem sap proteins: their identities and potential roles in the interaction between plants and phloem-feeding insects. J Exp Bot 57: 767-774.

27. Bradford MM (1976) A rapid and sensitive method for the quantitation of microgram quantities of protein utilizing the principle of protein-dye binding. Anal Biochem 72: 248-254.

28. Karimi M, Inzé D, Depicker A (2002) GATEWAYvectors for Agrobacteriummediated plant transformation. Trends Plant Sci 7: 193-195.

29. Clough SJ, Bent AF (1998) : Floral dip: a simplified method for Agrobacteriummediated transformation of Arabidopsis thaliana. Plant J 16: 735-743.

30. Ortego F, Novillo C, Castañera P (1996) Characterization and distribution of digestive proteases of the stalk corn borer, Sesamia nonagrioides Lef. (Lepidoptera: Noctuidae). Arch Insect Biochem Physiol 33: 163-180.

31. Shahnaz SN, van EJM, Damme E, Smagghe G (2008) Carbohydrate-binding activity of type-2 ribosome-inactivating protein SNA-I from elderberry (Sambucus nigra) is a determine factor for its insecticidal activity. Phytochem 69: 2972-2978.

32. Abbott WS (1925) A method of computing the effectiveness of an insecticide. J Econ Entomol 18: 265-267.

33. Carrillo L, Martinez M, Alvarez-Alfageme F, Castañera P, Smagghe G, et al. (2011a) A barley cysteine proteinase inhibitor reduces the performance of two aphid species in artificial diets and transgenic Arabidopsis plants. Transgenic Res 20: 305-319.

34. Lee MJ, Anstee JH (1995) Endoproteases from the midgut of larval Spodoptera littoralis include a chymotrypsin like enzyme with an extended binding site. Insect Biochem Molec Biol 25: 49-61.

35. Zwilling R, Medugorac I, Mella K (1972) The evolution of endopeptidases-XIV. Non-tryptic cleavage specificity of a baee-hydrolyzing enzyme ( $\beta$-protease) from Tenebrio molitor. Comp Biochem Physiol B 43: 419-424.

36. Bewley GC, DeVillez EJ (1968) Isolation and characterization of the digestive proteinases in the earthworm Lumbricus terrestris Linnaeus. Comp Biochem Physiol B 25: 1061-1066.

37. Malone LA, Burgess EPJ, Stefanovic D, Gatehouse HS (2000) Effects of four protease inhibitors on the survival of worker bumblebees, Bombus terrestris $\mathrm{L}$. Apidologie 31: 25-38.

38. Mulligan EA, Ferry N, Jouanin L, Romeis J, Gatehouse AMR (2010) Characterisation of adult green lacewing (Chrysoperla carnea) digestive physiology: impact of a cysteine protease inhibitor and a pyrethroid. Pest Manag Sci 66: 325-336.

39. Murdock LL, Brookhart G, Dunn PE, Foard DE, Kelley S, et al. (1987) Cysteine digestive proteinases in Coleoptera. Comp Biochem Physiol B 87: 783-787.

40. Novillo C, Castañera P, Ortego F (1997) Characterization and distribution of chymotrypsin-like and other digestive proteases in Colorado potato beetle larvae. Arch Insect Biochem Physiol 36: 181-201.
41. Carrillo L, Martinez M, Ramessar K, Cambra I, Castañera P, et al. (2011b) Expression of a barley cystatin gene in maize enhances resistance against phytophagous mites by altering their cysteine-proteases. Plant Cell Reports 30: 101-112.

42. Lampl N, Budai-Hadria O, Davydov O, Joss TV, Harrop SJ, et al. (2010) Arabidopsis AtSerpin 1, crystal structure and in vivo interaction with its target protease RESPONSIVE TO DESSICATION-21 (RD21). J Biol Chem 285: $13550-13560$

43. Al-Khunaizi M, Luke CJ, Cataltepe S, Miller D, Mills DR, et al. (2002) The serpin SON-5 is a dual mechanistic class inhibitor of serine and cysteine proteinases. Biochemistry 41: 3189-3199.

44. Brüning M, Lummer M, Bentele C, Smolenaars MMV, Rodenburg KW (2007) The Spn4 gene from Drosophila melanogaster is a multipurpose defence tool directed against proteases from different peptidase families. Biochem J 401: 325-331.

45. De Leo F, Bonadé-Bottino MA, Ceci LR, Gallerani R, Jouanin L (1998) Opposite effects on Spodoptera littoralis larvae of high expression level of a trypsin proteinase inhibitor in transgenic plants. Plant Physiol 118: 997-1004.

46. Marchetti S, Delledonne M, Fogher C, Chiaba C, Chiesa F, et al. (2000) Soybean Kunitz, C-II and PI-IV inhibitor genes confer different levels of insect resistance to tobacco and potato transgenic plants. Theor Appl Genet 101: 519-526.

47. Carlini CR, Grossi-de-Sá MF (2002) Plant toxic proteins with insecticidal properties. A review on their potentialities as bioinsecticides. Toxicon 40: 1515-1539.

48. Lara P, Ortego F, Gonzalez-Hidalgo E, Castañera P, Carbonero P, et al. (2000) Adaptation of Spodoptera exigua (Lepidoptera: Noctuidae) to barley trypsin inhibitor BTI-CMe expressed in transgenic tobacco. Transgenic Res 9: 169-178.

49. De Leo F, Bonade-Bottino M, Ceci LR, Gallerani R, Jouanin L (2001) Effects of a mustard trypsin inhibitor expressed in different plants on three Lepidopteran pests. Insect Biochem Mol Biol 31: 593-602.

50. Rahbé Y, Ferrasson E, Rabesona H, Quillien L (2003b) Toxicity to the pea aphid Acyrthosiphon pisum of anti-chymotrypsin of anti-chymotrypsin isoforms and fragments of Bowman-Birk protease inhibitors from pea seeds. Insect Biochem Mol Biol 33: 299-306.

51. Deraison C, Darboux I, Duportets L, Gorojankina T, Rahbé Y, Jouanin L (2004) Cloning and characterization of a gut-specific cathepsin L from the aphid Aphis gossypii. Insect Mol Biol 13: 165-177.

52. Cristofoletti PT, Ribeiro AF, Deraison C, Rahbé Y, Terra WR (2003) Midgut adaptation and digestive enzyme distribution in a phloem feeding insect, the pea aphid Acyrthosiphon pisum. J Insect Physiol 49: 11-24.

53. Rispe C, Kutsukake M, Doublet V, Hudaverdian S, Legeai F, et al. (2008) Large gene family expansion and variable selective pressures for cathepsin B in aphids. Mol Biol Evol 25: 5-17.

54. Rahbé Y, Deraison C, Bonade-Bottino M, Girard C, Nardon C, et al. (2003a) Effects of the cysteine protease inhibitor oryzacystatin (OC-I) on different aphids and reduced performance of Myzus persicae on OC-I expressing transgenic oilseed rape. Plant Sci 164: 441-450.

55. Azzouz A, Cherqui A, Campan EDM, Rahbé Y, Duport G, et al. (2005) Effects of plant protease inhibitors, oryzacystatin I and soybean Bowman-Birk inhibitor, on the aphid Macrosiphum euphorbiae (Homoptera, Aphididae) and its parasitoid Aphelinus abdominalis (Hymenoptera, Aphididae). J Insect Physiol 51: 75-86.

56. Cowgill SE, Wright C, Atkinson HJ (2002) Transgenic potatoes with enhanced levels of nematode resistance do not have altered susceptibility to nontarget aphids. Mol Ecol 11: 821-827.

57. Álvarez-Alfageme F, Martinez M, Pascual-Ruiz S, Castañera P, Diaz I, et al. (2007) Effects of potato plants expressing a barley cystatin on the predatory bug Podisus maculiventris via herbivorous prey feeding on the plant. Transgenic Res 16: $1-13$.

58. Ribeiro APO, Pereira EJC, Galvan TL, Picanco MC, Picoli EAT, et al. (2006) Effect of eggplant transformed with oryzacystatin gene on Myzus persicae and Macrosiphum euphorbiae. J Appl Entomol 130: 84-90. 